\title{
Generation of Nonclassical Biphoton States through Cascaded Quantum Walks on a Nonlinear Chip
}

\author{
Alexander S. Solntsev, ${ }^{1,}$ Frank Setzpfandt, ${ }^{2}$ Alex S. Clark, ${ }^{3}$ Che Wen Wu, ${ }^{1}$ Matthew J. Collins, ${ }^{3}$ Chunle Xiong, ${ }^{3}$ \\ Andreas Schreiber, ${ }^{4}$ Fabian Katzschmann, ${ }^{4}$ Falk Eilenberger, ${ }^{2}$ Roland Schiek, ${ }^{5}$ Wolfgang Sohler, ${ }^{4}$ \\ Arnan Mitchell, ${ }^{6}$ Christine Silberhorn, ${ }^{4}$ Benjamin J. Eggleton, ${ }^{3}$ Thomas Pertsch, ${ }^{2}$ \\ Andrey A. Sukhorukov, ${ }^{1}$ Dragomir N. Neshev, ${ }^{1}$ and Yuri S. Kivshar ${ }^{1}$ \\ ${ }^{1}$ Centre for Ultrahigh Bandwidth Devices for Optical Systems (CUDOS), Nonlinear Physics Centre, \\ Research School of Physics and Engineering, Australian National University, \\ Canberra 0200, Australian Capital Territory, Australia \\ ${ }^{2}$ Institute of Applied Physics, Abbe Center of Photonics, Friedrich-Schiller-Universität Jena, \\ Max-Wien-Platz, 1, 07743 Jena, Germany \\ ${ }^{3}$ CUDOS, the Institute of Photonics and Optical Science (IPOS), School of Physics, University of Sydney, \\ New South Wales 2006, Australia \\ ${ }^{4}$ Universität Paderborn, Warburger Strasse 100, 33098 Paderborn, Germany \\ ${ }^{5}$ University of Applied Sciences Regensburg, Prüfeninger Strasse 58, 93049 Regensburg, Germany \\ ${ }^{6}$ CUDOS, School of Electrical and Computer Engineering, RMIT University, \\ Melbourne, Victoria 3001, Australia \\ (Received 8 January 2014; revised manuscript received 12 May 2014; published 14 July 2014)
}

\begin{abstract}
We demonstrate a nonlinear optical chip that generates photons with reconfigurable nonclassical spatial correlations. We employ a quadratic nonlinear waveguide array, where photon pairs are generated through spontaneous parametric down-conversion and simultaneously spread through quantum walks between the waveguides. Because of the quantum interference of these cascaded quantum walks, the emerging photons can become entangled over multiple waveguide positions. We experimentally observe highly nonclassical photon-pair correlations, confirming the high fidelity of on-chip quantum interference. Furthermore, we demonstrate biphoton-state tunability by spatial shaping and frequency tuning of the classical pump beam.
\end{abstract}

DOI: 10.1103/PhysRevX.4.031007

Subject Areas: Optics, Quantum Physics

\section{INTRODUCTION}

Quantum systems allow us to access a multidimensional Hilbert space that lies beyond the realms of classical physics, facilitating the teleportation of quantum particles, unbreakable cryptographic schemes, and more powerful computer algorithms [1]. The most common way of encoding quantum information is a qubit, a quantum system with two possible states. Furthermore, it has been shown that quantum systems with more degrees of freedom allow for a reduction of the complexity of quantumprocessing schemes due to a smaller number of necessary information carriers [2]. Such multidimensional quantuminformation encoding has been used in trapped-ion quantum-computing architectures [3,4]. Optical quantum circuits also benefit from higher-dimensional qudits [5], and the necessary optical coherent control over several

\footnotetext{
*a.s.solntsev@gmail.com
}

Published by the American Physical Society under the terms of the Creative Commons Attribution 3.0 License. Further distribution of this work must maintain attribution to the author(s) and the published article's title, journal citation, and DOI. dimensions has already been demonstrated [6]. Multidimensional qudits based on photons can be effectively created through the process of spontaneous parametric down-conversion (SPDC) [7]. To manipulate these states, multiple beam splitters $[8,9]$ and integrated waveguide structures in the regime of quantum walks [10-15] have been employed. However, although linear optical circuits can effectively reshape the photon states and implement quantum computations, they cannot increase the total degree of entanglement present, characterized by the Schmidt number [16,17], when compared to the input quantum state.

Advanced quantum simulations, however, may require strongly nonclassical multidimensional states with largescale entanglement. In this work, we experimentally demonstrate, for the first time, to our knowledge, a nonlinear optical chip that enables the generation of nonclassical photon-pair states with a reconfigurable degree of entanglement in a multivalued discrete spatial coordinate. The entanglement is achieved through quantum interference of photon pairs generated by SPDC in a quadratic nonlinear waveguide array (WGA), where these photon pairs also undergo quantum walks. The combination of 
these two phenomena enables cascaded quantum walks that can be controlled by tuning the properties of the pump beam or changing the sample structure, providing a flexible tool for the preparation of states with a variable degree of nonclassicality in the spatial domain. This scheme inherently features the key benefits associated with integrated optical circuits, including the absence of propagation and incoupling losses and high interferometric stability. Hence, it is a promising photon source for flexible integrated quantum circuitry.

The remainder of this manuscript is organized in the following way. In Sec. II, we will explain the notion of cascaded quantum walks in detail. Experimental results characterizing the output state of cascaded quantum walks highlighting different tuning mechanisms are described in Secs. III and IV. Finally, we will discuss possible applications of our concept in Sec. V.

\section{CONCEPT OF CASCADED QUANTUM WALKS}

We consider the generation of photon pairs in a nonlinear WGA made from lithium niobate [18]. Because of the strong second-order optical nonlinearity of lithium niobate, photon pairs can be generated through SPDC of a shortwavelength pump beam. In comparison to the previously demonstrated biphoton generation in uncoupled waveguides or bulk crystals, our work offers unprecedented flexibility of biphoton-spatial-state control due to the unique properties of WGAs. Unlike single-mode waveguides, bulk nonlinear structures and nonlinear WGAs allow the generation of spatial entanglement; however, the spatial dispersion in the bulk is fundamentally different from waveguide arrays. In bulk structures, the rate of diffraction is proportional to the wavelength of light inside the material. In particular, the pump-beam diffraction is roughly one-half of the diffraction experienced by the neardegenerate signal and idler waves.

In contrast, in waveguide arrays, the rate of signal and idler diffraction is defined by the coupling, which can be flexibly engineered, for example, by varying the transverse waveguide separation. At the same time, the pump diffraction can be practically suppressed, allowing independent spatial manipulation of pump and photon pairs. For pump wavelengths around $775 \mathrm{~nm}$, as used in this study, strong localization of the corresponding waveguide mode confines the pump to the excited waveguide. Thus, photon pairs are continuously generated along the pump waveguide, as schematically illustrated in Fig. 1(a). The generated photons have approximately twice the wavelength of the pump beam, resulting in a larger optical waveguide mode and finite hopping probability between waveguides. As a result, the generated photon pairs undergo a quantum walk, which, due to interplay with the generation mechanism, shapes the correlations toward the output state [19].
In comparison to multimode nonlinear waveguides, our approach allows significantly more flexible control of the generated states by tuning the coupling between the waveguides or changing the properties of single waveguides.

Quantum walks have been studied in different systems, including trapped ions [20], atoms [21], and optics [22]. Because of their unique flexibility, precise control of system parameters, small decoherence, and the relative ease of transporting states over long distances, optical schemes provide an ideal platform for the implementation of quantum walks [10,12,15,23-25]. WGAs, periodic arrangements of waveguides allowing for photon hopping due to evanescent coupling of the optical modes of the individual waveguides [26], enable the scalability of quantum walks to a large number of positions and walkers. In a linear WGA, two-photon quantum walks of externally generated photons have been shown [10]. However, as mentioned earlier, quantum walks in linear systems can only reshape the nonclassical characteristics of an externally generated input, e.g., its entanglement. The total entanglement characterized by the Schmidt number $[16,17]$ can, at best, be preserved and will practically always decrease due to incoupling losses [13].

In contrast, the cascaded quantum walks introduced here allow for flexible control over the amount of entanglement as well as its particular distribution. The cascaded quantum walks in a waveguide array can be represented as a multilevel graph schematically shown in Fig. 1(b). Here, a linear quantum walk of the generated photon pair is visualized with a red two-dimensional (2D) grid, where $n_{s}$ and $n_{i}$ denote the positions of signal and idler photons. Each 2D grid represents a two-dimensional quantum walk, which is similar to the walks studied before in linear waveguide arrays [10], but in our case, the photons are generated by SPDC in the pumped waveguide. Starting from the pumped waveguide, photon pairs evolve over time by hopping between the waveguides. In an unperturbed WGA, photons have an equal probability of hopping to the left or right neighboring waveguides. In contrast to the previously studied quantum walks in linear structures, in our case, the pump beam (green) can initialize photon-pair quantum walks in the pumped waveguide at all possible distances $z$ from the input. This effect leads to the excitation of a cascade of interfering 2D quantum walks, visualized by the different layers of red grids in Fig. 1(b). Discrete red layers of quantum walks in Fig. 1(b) are only chosen for visualization purposes. Each of these quantum walks is a continuous-time quantum walk with propagation distance $z$ playing the role of time and changing from 0 to the total WGA length $L$. Because of quantum interference, different nodes along $z$ are combined into a $2 \mathrm{D}$ graph, resulting in a superposition of virtual 2D quantum walks originating from different positions. Such a superposition of quantum walks at different stages of their evolution is fundamentally 


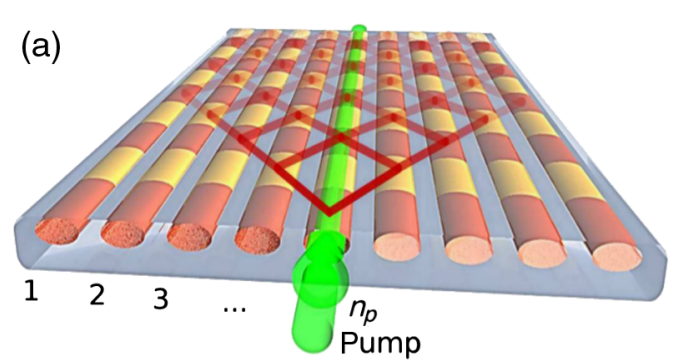

(c)

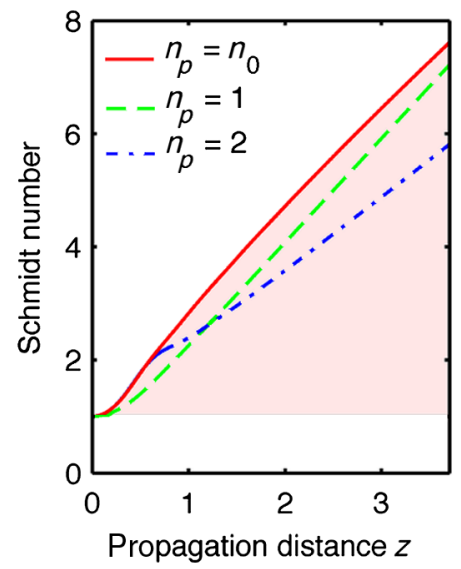

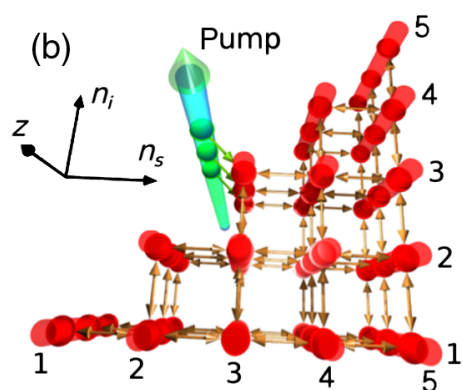

(d)

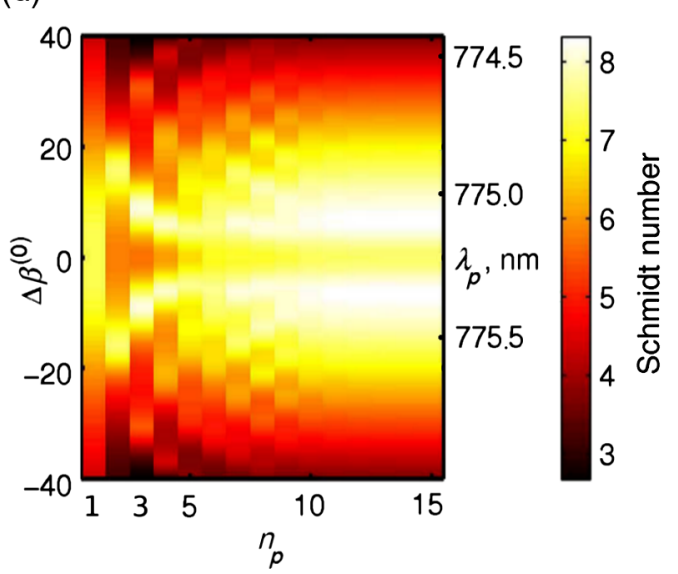

FIG. 1. (a) Schematic illustration of a quadratic nonlinear waveguide array. The pump (green) is coupled to the waveguide number $n_{p}$. The photon pairs (red) are generated in the pump waveguide and propagate in the regime of quantum walks. (b) Representation of a photon-pair quantum walk on a lattice, where $n_{s}$ and $n_{i}$ are the signal and idler photon positions, respectively. A pump (green arrow) continuously generates photon pairs along the propagation direction $z$; signal and idler photons walk between waveguides (orange arrows). (c) Photon-pair quantum-state Schmidt number versus the propagation distance $z$ normalized to the coupling length $\pi / C$ for zero phase mismatch $\Delta \beta^{(0)}=0$ and different pump sites: $n_{p}=n_{0}$ (red), $n_{p}=1$ (green), and $n_{p}=2$ (blue). A larger Schmidt number corresponds to higher spatial biphoton entanglement. (d) Schmidt number at the output of the waveguide array versus the phase mismatch $\Delta \beta^{(0)}$ corresponding to the pump wavelength $\lambda_{p}$ and versus the pumped waveguide number $n_{p}$ near the edge of the waveguide array.

impossible to achieve with any kind of linear device based on beam splitters without losses growing exponentially with the addition of each beam splitter.

The interference of virtual quantum walkers generated at different distances leads to the increase of entanglement. However, since, in general, SPDC produces photons of different wavelengths according to the energy-conservation law, entanglement may be generated in energy as well as spatial position [27]. Targeting spatial entanglement, in Figs. 1(c) and 1(d), we consider only degenerate SPDC where both photons have equal energy. With increasing propagation distance of the quantum walk, the spatial entanglement increases. The generation of entanglement is illustrated in Fig. 1(c), where we plot the numerically calculated Schmidt numbers [16,17] of the photon-pair state as a measure of the entanglement depending on the propagation distance for various pumped waveguides. Larger Schmidt numbers correspond to a higher degree of entanglement, while a Schmidt number equal to 1 corresponds to the absence of entanglement. We find an increasing Schmidt number for a range of input pump sites and note that different states can have the same Schmidt number. Cascaded quantum walks allow for easy tuning of the generated entanglement. First, the phase-velocity mismatch between the pump and the generated photon pairs can be changed, for example, by pump-wavelength tuning, thus influencing the generation of photon pairs. Second, the linear photon-pair quantum walks depend on the linear coupling in the WGA and thus can be controlled by changing the properties of the WGA. The engineering of WGAs allows for a multitude of different propagation regimes $[26,28,29]$. Here, we study the influence of the WGA edge as it demonstrates the effect of interrupting the structure periodicity. Figure 1(d) shows that the Schmidt number and thus the entanglement depend nontrivially on the single-waveguide phase mismatch $\Delta \beta^{(0)}$ and the pumpbeam position $n_{p}$ relative to the WGA edge. In the regime close to the degeneracy $\Delta \beta^{(0)}=0$, when the signal and idler photons have the same frequency and are thus indistinguishable, the quantum states emerging from the WGA are strongly spatially entangled, as indicated by large values of the Schmidt number. The generation of entanglement is a significant difference from the previously considered quantum walks in linear WGAs, where photons were already entangled at the input to obtain nontrivial statistics at the output [10]. 


\section{CONTROL OF QUANTUM STATES BY NONLINEAR PHASE MATCHING}

The highest degrees of entanglement are predicted for small degenerate phase mismatches, as shown in Fig. 1(d). Also, larger entanglement is expected for a quantum walk with a maximum number of spatial degrees of freedom. To achieve these conditions, we couple a pump beam of wavelength $\lambda_{p}=775.2 \mathrm{~nm}$ to waveguide $n_{0}$ in the center of our WGA, where the generated photon walkers travel ballistically across the WGA without interacting with the boundaries. Typical continuous-wave pump powers in all experiments reported here are below $0.5 \mathrm{~mW}$. Experimental measurements of the spatiospectral photon distributions and their correlations are performed using a custom-made spectrometer and a coincidence scheme. (See Appendixes B and C, respectively.)
The results of spatiospectrally resolved classical intensity measurements of the SPDC output are depicted in Fig. 2(a). They show one broad spectral maximum of the generated photon pairs around a wavelength of $2 \lambda_{p}=1550.4 \mathrm{~nm}$, very close to the wavelength of degenerate photon pairs, which is $1550.5 \mathrm{~nm}$. This wavelength also corresponds to the maximum transverse transport of the generated photons, as indicated by the broad intensity distribution. We note that the typical discrete diffraction pattern characteristic for ballistic transport phenomena [26] cannot be observed since photons are generated continuously along the WGA, as shown in Fig. 1(b).

To demonstrate the nonclassical nature of the generated photon walkers, biphoton correlation measurements are performed in the five central waveguides of the WGA after applying a narrow spectral filter rejecting the
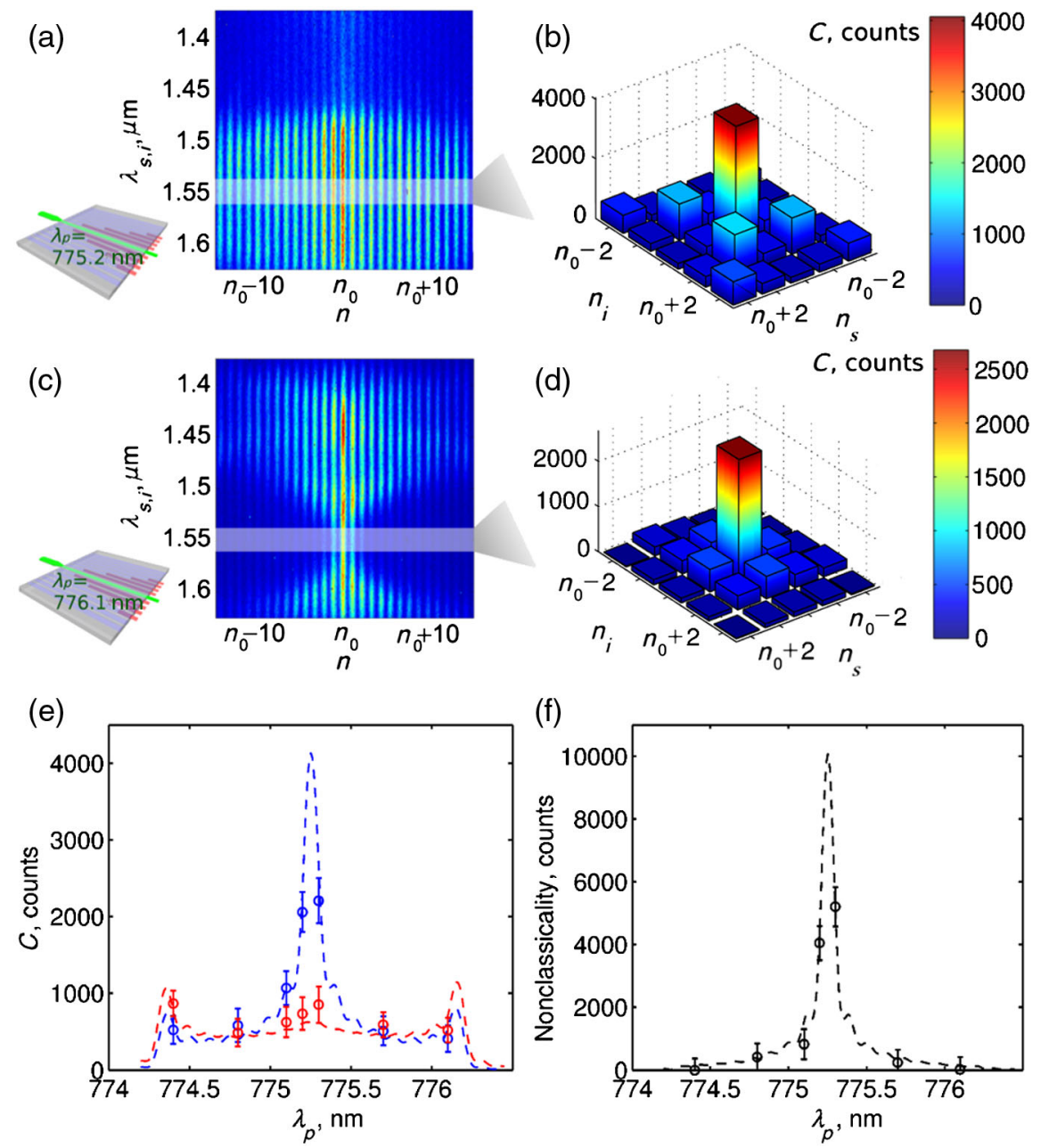

FIG. 2. (a),(c) Spatial-spectral photon-pair output distributions and (b),(d) corresponding correlations using a spectral filter (gray area). The pump beam is coupled to a waveguide at the center of the array $\left(n_{p}=n_{0}\right)$ with the pump wavelength (a), (b) $\lambda_{p}=775.2 \mathrm{~nm}$ and (c), (d) $\lambda_{p}=776.1 \mathrm{~nm}$. The long-wavelength part of the spectrum is not recorded due to a lack of sensitivity of the InGaAs camera. (e) Diagonal correlations characterizing simultaneous bunching and antibunching $\left\{n_{0}-1, n_{0}-1\right\}+\left\{n_{0}-1, n_{0}+1\right\}+\left\{n_{0}+1, n_{0}-1\right\}+$ $\left\{n_{0}+1, n_{0}+1\right\}$ (blue) and off-diagonal correlations $\left\{n_{0}, n_{0}-1\right\}+\left\{n_{0}-1, n_{0}\right\}+\left\{n_{0}+1, n_{0}\right\}+\left\{n_{0}, n_{0}+1\right\}$ (red) versus the pump wavelength $\lambda_{p}$ : theory (dashed lines) and experiment (circles with error bars). (f) Total nonclassicality versus the pump wavelength $\lambda_{p}$ : theory (dashed lines) and experiment (circles with error bars). 
nondegenerate photons. The resulting coincidence counts for the degenerate pairs are shown in Fig. 2(b), where the axis labels $n_{s}$ and $n_{i}$ refer to the output waveguides of indistinguishable photons, which we call signal $(s)$ and idler $(i)$ for convenience. Since photon pairs are generated in waveguide $n_{0}$ along the entire length of the array, the highest coincidence rate is always registered for position $\left\{n_{0}, n_{0}\right\}$, where both photons are in the pump waveguide. We observe that the probabilities of photons exiting either from the same waveguide, e.g., $\left\{n_{0}-1, n_{0}-1\right\},\left\{n_{0}, n_{0}\right\}$, $\left\{n_{0}+1, n_{0}+1\right\}$, or from the opposite waveguides, e.g., $\left\{n_{0}-1, n_{0}+1\right\},\left\{n_{0}+1, n_{0}-1\right\}$, are, in general, notably higher than other probabilities, namely, $\left\{n_{0}-1, n_{0}\right\}$, $\left\{n_{0}, n_{0}-1\right\},\left\{n_{0}+1, n_{0}\right\},\left\{n_{0}, n_{0}+1\right\}$. It is a sign for strong simultaneous photon bunching and antibunching and implies a nonclassical spatially entangled state $[19,30]$.

The experimental determination of the Schmidt number requires additional interference measurements to extract the phase structure of the photon-pair state, which is a challenging technical task. Therefore, to establish the nonclassicality of the photon correlations, we use the nonclassicality criterion in the form of the Bell-type inequality derived by Bromberg et al. [31] (see Appendix $\mathrm{G}$ below for a detailed mathematical definition) based on the analysis of the generalized Hong-Ou-Mandel effect. Our experiments showing the nonclassicality over 4 standard deviations above 0 demonstrate correlations that cannot be achieved with classical light. Whereas positive nonclassicality was observed for quantum walks in linear waveguide arrays [10,31], it required accurate coupling of indistinguishable photons to different waveguides at the input. In our work, a strongly nonclassical entangled biphoton state is generated even for a pump beam coupled to a single waveguide at the input, and, in particular, photon bunching and antibunching in the correlations appear to be more pronounced and clear compared to those achieved in linear waveguide arrays [10].

All presented results are in good agreement with numerical simulations, providing strong conjecture for the generation of a state with spatial entanglement over at least five waveguide positions. This conclusion is further reinforced by a theoretical analysis of the minimum Schmidt number necessary to produce the measured photon amplitudes. In this approach, we numerically investigate states in which the correlation amplitudes match the experimentally measured intensities but with randomly chosen phases. (See the last section of the Supplemental Material for details [32].) The smallest obtained Schmidt number is 2.2, providing further support for the existence of entanglement in our system. We note that our analysis is based on the assumption of pure biphoton states, without contributions from states with larger photon numbers. Considering the low-input pump powers, this assumption is well justified in our experimental system.

We further demonstrate the reconfigurability of the quantum states by changing the phase mismatch. As discussed above, Fig. 1(d) shows the predicted influence of the mismatch on the Schmidt number of the output state. With increasing absolute value of the mismatch, the Schmidt number, and hence the degree of entanglement, is decreasing. Experimentally, the mismatch is controlled by tuning the pump wavelength. In Fig. 2(c), we plot the classical SPDC output intensities for a pump wavelength of $\lambda_{p}=776.1 \mathrm{~nm}$. Clearly, the highest intensities are achieved for offset signal and idler wavelengths of 1450 and $1670 \mathrm{~nm}$, indicating nondegenerate SPDC. Exactly at these wavelengths, the photons exhibit maximum spatial broadening due to the constructive interference of the SPDC process. This effect results in an hourglass-shaped distribution of the generated photons, whose narrow waist is at the degenerate phase-matching wavelength. The results of correlation measurements for this pump wavelength are presented in Fig. 2(d). We again find a dominating contribution from the $\left\{n_{0}, n_{0}\right\}$ position. However, in contrast to the case described above, no pronounced bunching and antibunching in other waveguides could be observed. Such output states can be reproduced with a classical walker, as indicated by zero nonclassicality. All results are in excellent agreement with our theoretical modeling. (See the Supplemental Material for comparison between theory and experiment [32].)

To test the tunability of the quantum states, we conduct correlation measurements for seven different pump wavelengths. Summarizing the results of these experiments, Fig. 2(e) shows the sum of the correlation counts in the diagonal positions $\left\{n_{0}-1, n_{0}-1\right\},\left\{n_{0}+1, n_{0}+1\right\}$, $\left\{n_{0}-1, n_{0}+1\right\}$, and $\left\{n_{0}+1, n_{0}-1\right\}$ with the blue circles and in the off-diagonal positions $\left\{n_{0}-1, n_{0}\right\}$, $\left\{n_{0}, n_{0}-1\right\},\left\{n_{0}+1, n_{0}\right\}$, and $\left\{n_{0}, n_{0}+1\right\}$ with the red circles. The corresponding dashed lines are numerical results. For pump wavelengths close to the degenerate phase matching at $\lambda_{p}=775.25 \mathrm{~nm}$, the count numbers on the diagonal positions of the correlation matrix are notably larger than the counts on the off diagonals. It indicates bunching and antibunching of photons, as discussed above. Such behavior cannot be achieved with classical walkers, as is shown by the corresponding values of the nonclassicality in Fig. 2(f). The experimental nonclassicality attains values over 4 times higher than the standard deviation near the degenerate phase-matching wavelength. These results illustrate that the tunability of the quantum walk and the control over the emerging spatial correlations can be beneficial for reconfigurable quantum simulations based on quantum walks.

\section{CONTROL OF QUANTUM STATES BY QUANTUM REFLECTION FROM A DEFECT}

In this section, we show that the output photon statistics can be controlled by the engineering of structural defects in the array. Such defects lead to the interference of incident 


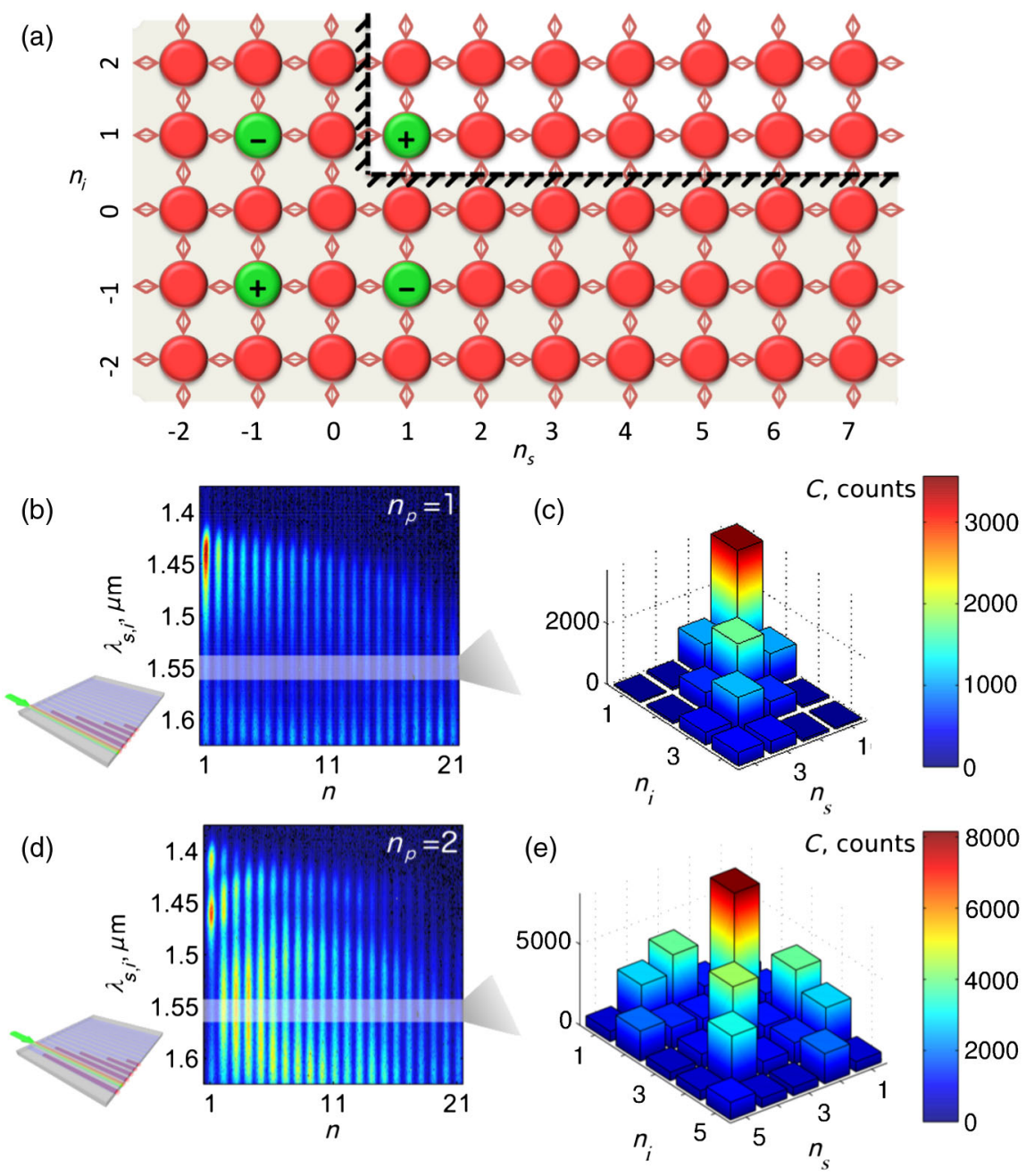

FIG. 3. (a) Lattice representation of the photon-pair states. Shading marks the image space beyond the array boundary, and green color with plus and minus signs marks the locations of real and virtual pump sources of biphotons. (b),(d) Spatial-spectral photon-pair output distributions and (c),(e) corresponding correlations using a spectral filter (gray area). (b),(c) The pump wavelength is $\lambda_{p}=775.3 \mathrm{~nm}$, and the pump is coupled to the first waveguide from the edge $\left(n_{p}=1\right)$. (d),(e) The pump wavelength is $\lambda_{p}=775.7 \mathrm{~nm}$, and the pump is coupled to the second waveguide from the edge $\left(n_{p}=2\right)$. Different pump wavelengths are selected to achieve maximally nonclassical states.

and reflected photons from the defect. As a simple example of a structural defect, we consider the edge of the WGA, which is accessed by placing the pump beam closer to the WGA boundary. Hence, the real photon positions are restricted to $n_{s, i} \geq 1$. Accordingly, the quantum walk is truncated. The accessible part of the quantum walk is represented by the white area in Fig. 3(a). The photon pairs are generated through SPDC at the position of the pump, marked with a green color and a plus sign. The example in Fig. 3(a) corresponds to the pump coupled to the first waveguide, with $n_{s}=n_{i}=n_{p}=1$. To describe the quantum walks at the reflecting boundary, we use an approach similar to the method of charge images in electrostatics [33] and formally extend the two-dimensional lattice representation of the quantum walk $[10,30]$ beyond the boundary $\left(n_{s, i} \leq 0\right)$, as marked with gray shading in Fig. 3(a). Three additional virtual sources appear in the imaginary space, which are in phase or out of phase with the real source, as marked with plus and minus symbols. Remarkably, the outof-phase virtual sources are positioned on the antidiagonal, where $n_{s}=-n_{i}= \pm n_{p}$, whereas real sources can only appear on a diagonal. Accordingly, the interference between the quantum walkers, i.e., photon pairs generated by real and virtual sources, gives rise to unique photon-pair correlations that cannot be simply achieved with real sources away from the WGA edge.

Experimentally obtained photon-pair spectral-spatial distributions and corresponding correlation measurements for two different distances of the pump waveguide from the edge are shown in Figs. 3(b)-3(e). Both measurements are conducted close to the respective wavelengths of degenerate phase matching, which depends on the distance from 
the WGA edge, leading to different pump wavelengths in the presented experiments. Because of reflections from the WGA boundary, interference fringes are observed in the photon-pair spectral-spatial distributions [Figs. 3(b) and 3(d)]. Accordingly, the photon correlations strongly depend on the pump position away from the edge waveguide, being formed by interference of photons reflected from the edge and photons propagating unobstructed. We note that whereas the spatial correlations in the WGA center have fourfold symmetry [see Figs. 2(b) and 2(d)], only twofold symmetry due to the photon indistinguishability remains close to the edge, as seen in Figs. 3(c) and 3(e). Because of the boundary, the antibunching of photons is no longer possible for coupling to the first waveguide; however, we still observe a strong bunching. For pump coupling to the second waveguide, we measure larger probabilities for bunching and symmetric antibunching. Experimental nonclassicalities are calculated to be 2440 counts for coupling to the first waveguide and 3600 counts for coupling to the second waveguide. Both values are over 3 standard deviations above 0 . Thus, we show that generated nonclassical quantum states can be reshaped by the utilization of structural defects. (See the Supplemental Material for more cases and the analysis of nonclassicality [32].)

\section{DISCUSSIONS}

The experimental results shown above were obtained using periodically poled waveguides, having some inhomogeneities in the poling domain sizes. Nevertheless, a good agreement with numerical simulations demonstrates that the operation is robust with respect to poling. We have additionally performed independent experiments with WGA samples without poling and operating at a shorter pump wavelength (see the Supplemental Material [32]), confirming the broad applicability of the developed approaches to various nonlinear integrated platforms.

While we have characterized biphoton correlations at the WGA output, our platform is well suited for the generation of multiple photon pairs along the WGA length, which would produce highly nonclassical multiphoton correlations. Such multiphoton correlations can also be manipulated by adjusting the phase-matching conditions for SPDC or changing the spatial distribution of the pump beam. Additional flexibility can also be achieved through specially designed poling patterns.

Because of the qualitatively new physics and control mechanisms explained in the previous sections, our scheme has the potential to be used for the generation of large-scale spatial entanglement with precisely controllable spatial correlations. The large number of discrete reconfigurable spatial modes makes this system a prime candidate for the generation of spatial qudits and cluster states, whereas previous bulk-optics approaches aimed at qudit and cluster-state generation are either difficult to scale [34] or produce only approximate analogues of the required states [35].

This work is also closely related to active research on the applications of optical quantum walks [10,12,13,15,23-25] for efficient quantum simulations. The key to unraveling their full potential is on-chip integration that enables better scalability to a large number of positions and walkers in comparison to bulk optical schemes. However, in all optical quantum-walk schemes to date, the correlated photons were generated externally to the WGA, which could introduce quantum decoherence and impose stringent requirements on the coupling losses associated with the connection of the WGA to the photon sources. In this work, we have experimentally demonstrated the concept of an integrated quantum circuit with photon sources, where a nonlinear WGA is used for both photon-pair generation and quantum walks. This approach may enable better scalability of the number of walkers within a quantum photonic circuit. In future works, it will be important to explore what types of quantum simulations may be carried out by integrated quantum walks on a nonlinear chip. Another interesting research direction would be to determine how introducing inhomogeneous coupling between the waveguides or inhomogeneous phase matching could help to achieve ultimate flexibility in generating tailored correlations and entanglement.

Finally, we believe that our work may have broad implications for other areas of physics. In our manuscript, we show that utilizing large-scale quantum interference enabled by nonlinearity in spatially nontrivial systems may allow novel operating regimes and enhanced control. Similar ideas may be utilized in a range of physical systems. For example, our previous theoretical research indicates that integrated biphoton generation and quantum walks may also be facilitated by spontaneous four-wave mixing, which is an analogue of SPDC in schemes with cubic nonlinearity, such as siliconbased devices [36]. Outside of the optical community, our approach may be realized in Bose-Einstein condensates by combining four-wave-mixing-generated pair-correlated atoms [37] and periodic lattices [38].

\section{ACKNOWLEDGMENTS}

We acknowledge Gerard Milburn, Andrew White, Andreas Christ, and Regina Kruse for useful discussions and Yoo Hong Min for help with sample fabrication. This work was supported by the Discovery Projects (DP1093767 and DP130100135), Future Fellowship (FT100100160), Laureate Fellowship (FL120100029), Centre of Excellence (CE110001018), and Discovery Early Career Researcher Grants (DE130101148 and DE120100226) programs of the Australian Research Council (ARC), Project No. IST-2000-26005 (ROSA) of the European Commission, and the Thuringian Ministry of Education and Research (Project MeMa). 


\section{APPENDIX A: WAVEGUIDE-ARRAY SAMPLE}

These Appendixes include a detailed description of our experimental methods and theoretical models. The WGAs are fabricated in $z$-cut $\mathrm{LiNbO}_{3}$ wafers by titanium indiffusion. The domain inversion is achieved by electric field poling. The WGA used for the experiments presented here consists of 101 waveguides with a spacing of $13.5 \mu \mathrm{m}$ and a length of $51 \mathrm{~mm}$. (See the Supplemental Material for the WGA characterization [32].) The coupling length between the neighboring waveguides is found to be $\gg 51 \mathrm{~mm}$ at the pump wavelength and about $12 \mathrm{~mm}$ at the biphoton wavelengths.

\section{APPENDIX B: MEASUREMENT OF SPECTRAL-SPATIAL SPONTANEOUS PARAMETRIC DOWN-CONVERSION DISTRIBUTIONS}

The schematic illustration of the experimental setup is shown in Fig. 4. Our goal is to measure the spectral-spatial SPDC intensity output of the $\mathrm{Ti}: \mathrm{LiNbO}_{3}$ WGAs. The sample is heated to a temperature of approximately $240{ }^{\circ} \mathrm{C}$ to phase match the type-I ( $e e-e$ corresponding to extraordinary signal, idler, and pump polarizations) SPDC process and to avoid photorefractive effects. A single waveguide of the WGA is excited by a tunable $\mathrm{cw}$ laser operating around $\lambda_{p} \approx 775 \mathrm{~nm}$. At the pump wavelength, the WGA is multimoded; however, by carefully aligning the input beam, we ensure predominant coupling of the input beam to the fundamental $\mathrm{TM}_{00}$ mode. This mode does not exhibit evanescent coupling to the neighboring waveguides and hence is bound to the excitation site. In contrast, the longerwavelength SPDC photon pairs could propagate across the WGA.

After the crystal, the pump beam is filtered out by a longpass filter with a cutoff wavelength of approximately $1 \mu \mathrm{m}$; then, the SPDC output is refracted by a prism, which allows us to study the SPDC distribution in different waveguides for various frequency mismatches $\Delta \omega$. Thus, we obtain the spectral-spatial SPDC distribution $I(n, \lambda)$ that is measured by a cooled IR camera. The spectral resolution is approximately $30 \mathrm{~nm}$.

\section{APPENDIX C: MEASUREMENT OF PHOTON-PAIR CORRELATIONS}

The schematic representation of the SPDC quantum correlation measurement setup is shown in Fig. 5. Similar

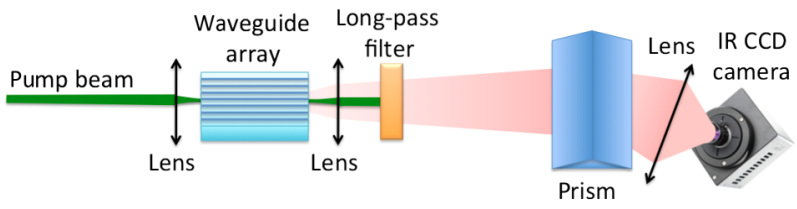

FIG. 4. Schematic illustration of the experimental setup of the spectral-spatial SPDC intensity-distribution measurements.

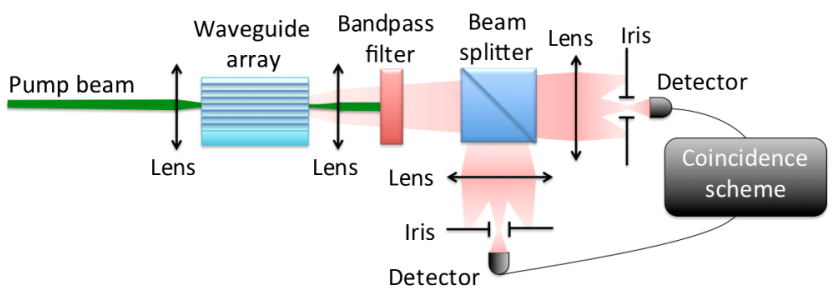

FIG. 5. Schematic illustration of the experimental setup for the SPDC photon-pair correlation measurements.

to the spectral experiments, a single waveguide of the WGA is excited by a tunable $\mathrm{cw}$ laser operating around $\lambda_{p} \approx 775 \mathrm{~nm}$ to phase match the type-I $(e e-e)$ spontaneous parametric down-conversion process, while the sample is heated to a temperature of approximately $240^{\circ} \mathrm{C}$. The pump power is kept below $0.5 \mathrm{~mW}$ to minimize photorefraction.

Experimentally, we measure the photon-pair correlations for a range of pump wavelengths on two cooled InGaAs single-photon detectors (IDQuantique 210) after passing through a 12-nm broad-bandpass filter with a center wavelength of $1550 \mathrm{~nm}$ used for the selection of degenerate photon pairs. One arm is detected in a free-running mode (master), the output of which triggers the second in a gated mode (slave). A beam splitter is used to separate two photons of a pair with 50\% efficiency, while irises allow for individual waveguide-output selection. This scheme allows us to directly measure photon-pair correlations as defined in Ref. [31].

The photon arrival times in both detectors are recorded with a two-channel event counter (PicoQuant HydraHarp 400 , timing resolution $2 \mathrm{ps}$ ). To retrieve the number of two-photon coincidences, the delay between consecutive events in the master and slave detectors is calculated, and two-photon events are sorted into time bins of 50-ps width, according to this delay. A characteristic plot of the number of events versus the delay is shown with the blue symbols in Fig. 6. A pronounced maximum is a clear sign for coincidences. The number of coincidences $\Gamma_{n_{s}, n_{i}}^{\mathrm{raw}}$ is determined by fitting a Gaussian function to the measured counts (red line) and calculating the area under the function

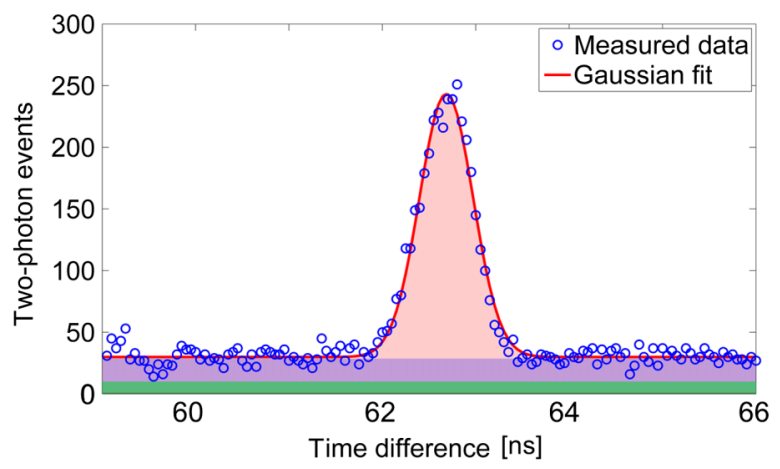

FIG. 6. Coincidences versus time delay between single-photon detectors: biphoton coincidences (red), events from uncorrelated photons (blue), and dark counts (green). 
(red area). Coincidences for every waveguide combination and pump wavelength or position are integrated for $10 \mathrm{~min}$.

After the measurement, we perform a renormalization, taking into account fluctuations in the coupling efficiency from waveguides to single-photon detectors as follows:

$$
\Gamma_{n_{s}, n_{i}}=\frac{\Gamma_{n_{s}, n_{i}}^{\mathrm{raw}}}{\chi_{n_{s}, n_{i}}^{\text {mater }} \varkappa_{n_{s}, n_{i}}^{\text {slave }}} .
$$

Here, $\Gamma_{n_{s}, n_{i}}^{(\mathrm{raw})}$ are raw coincidences measured by the coincidence scheme, and $\varkappa_{n_{s}, n_{i}}^{\text {master }}$ and $\varkappa_{n_{s}, n_{i}}^{\text {slave }}$ are variations in waveguide-to-detector coupling due to switching between different waveguides and experimental fluctuations over the course of the experiment. These coupling-variation coefficients are calculated as follows. Ideally, moving the slave detector should not affect master-detector single counts. In real experimental settings, the mechanical adjustment required for switching between waveguide pairs $\left(n_{s}, n_{i}\right)$ leads to variations in the coupling efficiencies and hence the single counts. To compensate for this effect, we calculate the master-detector coupling variation:

$$
\varkappa_{n_{s}, n_{i}}^{\text {master }}=\frac{N_{n_{s}, n_{i}}^{\text {master }}-N^{\text {master, dark }}}{\frac{1}{n} \sum_{n_{i}}\left(N_{n_{s}, n_{i}}^{\text {master }}-N^{\text {master, dark }}\right)} .
$$

Here, $N_{n_{s}, n_{i}}^{\text {master }}$ is the number of master-detector single counts, $N^{\text {master, dark }}$ is the number of master-detector dark counts, and $n$ is the number of measured WGA outputs.

Similarly, we compensate for the slave-detector coupling variation. Here, we have to take into account that the slave detector can register an event only after it has been triggered by the master detector. Hence, the coupling variation has to be normalized to the number of master trigger events. Furthermore, three different types of events are detected in the slave detector: coincidences, single photons not related to a photon pair, and dark counts. They are schematically shown by the red, blue, and green areas in Fig. 6. From these events, only the single-photon counts that are not related to a pair depend exclusively on the slave-channel number $n_{i}$; hence, they are used for the normalization. Since the efficiency of collecting and detecting a photon is smaller than 0.03 , the slave-detector coupling variation is calculated as follows:

$$
\varkappa_{n_{s}, n_{i}}^{\text {slave }}=\frac{N_{n_{s}, n_{i}}^{\text {slave }}-N^{\text {slave,dark }}-N_{n_{s}, n_{i}}^{\text {coinc }}}{\frac{1}{n} \sum_{n_{s}}\left(N_{n_{s}, n_{i}}^{\text {slave }}-N^{\text {slave,dark }}-N_{n_{s}, n_{i}}^{\text {coinc }}\right)} .
$$

Here, $N_{n_{s}, n_{i}}^{\text {slave }}$ is the number of slave-detector single counts, $N^{\text {slave,dark }}$ is the number of slave-detector dark counts, and
$N^{\text {coinc }}$ is the number of coincidence counts calculated by fitting temporary resolved coincidence data; see Fig. 6. This fitting also allows us to calculate the statistical errors.

We note that in this experiment, the measured correlations should be symmetric in respect to signal and idler photons due to their indistinguishability, i.e., $\Gamma_{n_{s}, n_{i}}=\Gamma_{n_{i}, n_{s}}$. Since, in the experiment, we arbitrarily assign "signal" and "idler" labels to our detectors, a meaningful measure of correlations would be the average symmetrized value $\Gamma_{n_{s}, n_{i}}^{\mathrm{sym}}=\left(\Gamma_{n_{i}, n_{s}}+\Gamma_{n_{s}, n_{i}}\right) / 2$. We use $\Gamma_{n_{s}, n_{i}}^{\mathrm{sym}}$ both for correlations and for nonclassicality plots.

\section{APPENDIX D: MODEL}

We consider a quadratic nonlinear process-spontaneous parametric down-conversion-and study the generation of photon pairs with the subsequent quantum walks in an array of coupled quadratic nonlinear waveguides, or a waveguide array. We analyze type-I SPDC, when a pump beam generates signal and idler photons of the same polarization. The quantum walks of generated photon pairs in the WGA can occur due to photon tunneling between waveguides, and we consider a common case when such tunneling occurs between neighboring waveguides. The tunneling rate is characterized by the coupling coefficients $C_{s}$ and $C_{i}$ for the signal and idler photons, respectively $[10,19,39]$.

For a continuous-wave pump at frequency $\omega_{p}$, the signal and idler photons will be generated with frequencies satisfying the energy-conservation relation $\omega_{s}+\omega_{i}=\omega_{p}$. We consider near-degenerate SPDC, when phase matching is achieved for photons with frequencies approximately half of the pump frequency. Then, we analyze a frequency range close to degeneracy, where the coupling coefficients are essentially the same for the signal and idler photons, and denote $C_{s, i} \equiv C$. For a pump beam with optical frequency $\omega_{p} \simeq 2 \omega_{s, i}$, the corresponding coupling coefficient $C_{p}$ would generally have a much smaller value compared to the signal and idler waves $C_{p} \ll C$ due to the weaker mode overlap between neighboring waveguides at higher frequencies [39]. In our experimental samples, $C_{p} L \ll 1$, where $L$ is the WGA length, and therefore the coupling effects can be neglected for the pump beam $\left(C_{p} \approx 0\right)$. Then, in the undepleted pump regime, the input pump-beam profile amplitude $E_{n}^{(p)}$ remains constant inside the WGA. Accordingly, the evolution of photon pairs with frequencies $\left(\omega_{s}, \omega_{i}\right)$ is governed by the Hamiltonian accounting for linear photon tunneling between the waveguides [10] and photon generation through SPDC [40] $\hat{H}=\hat{H}^{(\text {tun })}+\hat{H}^{\text {(SPDC) }}$ :

$$
\begin{aligned}
\hat{H}^{\text {(tun })}= & \hbar \sum_{n_{s}}\left\{\left[\beta_{s}\left(\omega_{s}\right)-\beta_{p} / 2\right] \hat{a}_{n_{s}}^{\dagger} \hat{a}_{n_{s}}+C \hat{a}_{n_{s}-1}^{\dagger} \hat{a}_{n_{s}}+C \hat{a}_{n_{s}+1}^{\dagger} \hat{a}_{n_{s}}\right\} \\
& +\hbar \sum_{n_{i}}\left\{\left[\beta_{i}\left(\omega_{i}\right)-\beta_{p} / 2\right] \hat{a}_{n_{i}}^{\dagger} \hat{a}_{n_{i}}+C \hat{a}_{n_{i}-1}^{\dagger} \hat{a}_{n_{i}}+C \hat{a}_{n_{i}+1}^{\dagger} \hat{a}_{n_{i}}\right\}+\text { H.c., }
\end{aligned}
$$




$$
\hat{H}^{(\mathrm{SPDC})}=i \hbar \gamma \sum_{n_{p}} E_{n_{p}}^{(p)} \hat{a}_{n_{s}}^{\dagger} \hat{a}_{n_{i}}^{\dagger} \delta_{n_{s}, n_{p}} \delta_{n_{i}, n_{p}}+\text { H.c. }
$$

Here, $\hat{a}^{\dagger}$ and $\hat{a}$ are creation and annihilation operators of photons with frequencies $\left(\omega_{s}, \omega_{i}\right), \beta_{s}$ and $\beta_{i}$ are signal and idler propagation constants, $n_{s}$ and $n_{i}$ are the waveguide numbers describing the positions of the signal and idler photons, $E_{n}^{(p)}$ is the pump amplitude in waveguide number $n_{p}$, and $\gamma$ is a nonlinear coefficient. We take into account that propagation constants $\beta_{s}$ and $\beta_{i}$ can depend strongly on the signal and idler frequencies $\omega_{s}$ and $\omega_{i}$, whereas we neglect the weak dependence of $C$ and $\gamma$ on frequency close to degeneracy.

If we assume that there is no decoherence, then the generation of photon pairs in quadratic nonlinear WGAs through SPDC in the absence of multiple photon pairs can be characterized by a pure biphoton state with wave function $\psi_{n_{s}, n_{i}}$, whose evolution is governed by a Schrödinger-type equation [30]:

$$
\begin{aligned}
\frac{d \psi_{n_{s}, n_{i}}(z, \omega)}{d z}= & i C\left[\psi_{n_{s}-1, n_{i}}(z, \omega)+\psi_{n_{s}, n_{i}-1}(z, \omega)+\psi_{n_{s}+1, n_{i}}(z, \omega)+\psi_{n_{s}, n_{i}+1}(z, \omega)\right] \\
& +i \Delta \beta^{(0)}(\omega) \psi_{n_{s}, n_{i}}(z, \omega)+\gamma E_{n_{s}}^{(p)} \delta_{n_{s}, n_{i}}
\end{aligned}
$$

where, to simplify the notation, we denote the signal frequency as $\omega_{s}=\omega$, while the idler frequency is defined by the pump and signal frequencies as $\omega_{i}=\omega_{p}-\omega_{s}$. The variable $z$ denotes the propagation direction along the WGA, and $\delta$ is the Kronecker delta function. Compared to a $k$-space representation [19], this formalism is more convenient for modeling photon-pair generation and quantum walks at the edge of a nonlinear WGA. The singlewaveguide phase mismatch $\Delta \beta^{(0)}$ is calculated, assuming a homogenous temperature distribution along the waveguide and ideal periodic poling using the refractive-index data for bulk $\mathrm{LiNbO}_{3}$ :

$$
\begin{aligned}
\Delta \beta^{(0)}= & \omega_{p} n^{p}\left(\omega_{p}, T\right) / c-\omega n^{s}(\omega, T) / c \\
& -\left[\omega_{p}-\omega\right] n^{i}\left(\omega_{p}-\omega, T\right) / c-2 \pi / L_{p} .
\end{aligned}
$$

Here, $n^{(p, s, i)}$ is an extraordinary or ordinary bulk refractive index [41], depending on the sample configuration; $T$ is the WGA temperature, and $L_{p}$ is a poling period in the case of a periodically poled WGA.

\section{APPENDIX E: CALCULATION OF EXPERIMENTALLY OBSERVABLE CHARACTERISTICS}

After calculating the wave function, we obtain twophoton correlations in real space as $\Gamma_{n_{s}, n_{i}}(\omega)=$ $\left|\psi_{n_{s}, n_{i}}(L, \omega)\right|^{2}$, where $L$ is the propagation length. Note that since the signal and idler photons have the same polarization and correspond to the same modes of individual waveguides, there is a symmetry relation $\Gamma_{n_{s}, n_{i}}\left(\omega_{s}\right) \equiv \Gamma_{n_{s}, n_{i}}\left(\omega_{i}\right)$.

In the spectral experiment, we measure the spectralspatial SPDC intensity at the output of a $\mathrm{LiNbO}_{3}$ WGA. To calculate the spectral-spatial output, we sum the probabilities of photons arriving to detector $n$ as follows:

$$
I(n, \omega)=\sum_{n_{s}} \Gamma_{n_{s}, n_{i}}(\omega) \delta_{n_{i}, n}+\sum_{n_{i}} \Gamma_{n_{s}, n_{i}}(\omega) \delta_{n_{s}, n} .
$$

Since, in the experiment, we resolve the wavelength with a prism, we convert frequency to wavelength $\lambda=2 \pi c / \omega$, thus obtaining the output spectral-spatial distribution $I(n, \lambda)$. Then, we simulate the spatial output profile by approximating the individual waveguide modes as almost nonoverlapping Gaussians $I(x, \lambda)=\sum_{n} I(n, \lambda) \exp$ $\left[-\left(x-d_{w} n\right)^{2} / w^{2}\right]$, where $d_{w}=13.5 \mu \mathrm{m}$ is the spacing between the centers of the waveguides and $w=3.5 \mu \mathrm{m}$ is the beam width. To emulate the limited spectral resolution due to the finite-mode diameter in the direction resolved by a prism, we also smooth the wavelength axis with strongly overlapping Gaussians $\tilde{I}(x, \lambda)=$ $\int d \lambda_{1} I\left(x, \lambda_{1}\right) \exp \left[-\left(\lambda-\lambda_{1}\right)^{2} / r^{2}\right]$, where $r=30 \mathrm{~nm}$ is the experimental wavelength-resolution parameter. We compare the spectral-spatial output $\tilde{I}(x, \lambda)$ with the experiment.

Two-photon correlation measurements are performed experimentally using a narrow-band spectral filter that transmits only the wavelengths close to degeneracy. The photon correlation at the filter output is

$$
\Gamma_{n_{s}, n_{i}}^{(F)}=\int d \omega_{s} F\left(\omega_{s}\right) F\left(\omega_{p}-\omega_{s}\right)\left|\psi_{n_{s}, n_{i}}\left(L, \omega_{s}\right)\right|^{2} .
$$

Here, $F(\omega)$ is a filter-transmission spectrum. For narrow filtering, the resulting correlation function will be simply proportional to $\Gamma_{n_{s}, n_{i}}\left(\omega_{p} / 2\right)$. Following filtering, the photons pass through a beam splitter, and then the spatial positions are selected by slits. For the measurement of photons at different spatial locations, with $n_{s} \neq n_{i}$, the detection probability is $\Gamma_{n_{s}, n_{i} \neq n_{s}}^{(D)}=(1 / 2)^{2}\left[\Gamma_{n_{s}, n_{i}}^{(F)}+\Gamma_{n_{i}, n_{s}}^{(F)}\right]$, where we take into account that $(1 / 2)$ is the probability that a photon from a particular waveguide passes through the beam splitter in the direction of the correspondingly aligned slit, and the detectors do not distinguish between the signal and idler photons. For the measurement of photons at the 
same spatial location, with $n_{s}=n_{i}$, the detection probability is $\Gamma_{n_{s}, n_{s}}^{(D)}=(1 / 2) \Gamma_{n_{s}, n_{s}}^{(F)}$, where $(1 / 2)$ is the probability that the photons will go in different directions after the beam splitter. Taking into account that $\Gamma_{n_{i}, n_{s}}^{(F)} \equiv \Gamma_{n_{s}, n_{i}}^{(F)}$, we conclude that for any photon position, the detection probability is

$$
\Gamma_{n_{s}, n_{i}}^{(D)}=(1 / 2) \Gamma_{n_{s}, n_{i}}^{(F)}
$$

\section{APPENDIX F: SCHMIDT DECOMPOSITION}

We numerically study the Schmidt decomposition of the wave function at degeneracy $\psi_{n_{s}, n_{i}}\left(L, \omega_{p} / 2\right)$ defined as follows [42]:

$$
\psi_{n_{s}, n_{i}}\left(L, \omega_{p} / 2\right)=\sum_{m=1}^{N} \sqrt{\Lambda_{m}} \phi_{m, n_{s}}^{(1)} \phi_{m, n_{i}}^{(2)} .
$$

Here, $N$ equals the minimum number of possible values of $n_{s}$ or $n_{i}$ (the number of waveguides in the WGA). The orthonormal sets of vectors $\phi_{m}^{(1)}$ and $\phi_{m}^{(2)}$ are called Schmidt modes. The non-negative weight factors $\Lambda_{m}$, called Schmidt coefficients, satisfy the normalization condition $\sum_{m=1}^{N} \Lambda_{m}=1$.

The number of nonzero Schmidt coefficients $\Lambda_{m}$ in the decomposition is called the Schmidt rank and is usually less than $N$. In the particular case of only one nonzero Schmidt coefficient, the state $\psi_{n_{s}, n_{i}}$ is factorized as a product of two Schmidt modes, which means that the degrees of freedom $n_{s}$ and $n_{i}$ are not entangled. When the Schmidt rank is more than 1 , then there is more than one summand in (F1) and the degrees of freedom are entangled.

Another quantitative measure of entanglement is the Schmidt number [16] or a cooperativity parameter [17] that characterizes the degree with which different Schmidt modes are excited:

$$
K=\frac{1}{\sum_{m} \Lambda_{m}^{2}}
$$

Larger Schmidt numbers correspond to a higher degree of entanglement.

In this work, we do not perform phase-related measurements, so we cannot experimentally reconstruct the Schmidt decomposition. However, assuming pure states, we can determine a lower bound of experimentally achieved entanglement using stochastic methods. Based on the experimental coincidence measurements, we have calculated that the Schmidt number for correlations in Figs. 2(b), 2(d), 3(c), and 3(e) is bounded by a minimum value of 2.2, which confirms the presence of spatial entanglement. We have also demonstrated that the minimal bound on the Schmidt number is higher when biphoton spatial correlations feature antibunching. (See the Supplemental Material [32].)

\section{APPENDIX G: NONCLASSICALITY}

For classical light, the diagonal correlations $\Gamma_{n_{s}, n_{s}}$ or $\Gamma_{n_{i}, n_{i}}$ are related to correlations in the off-diagonal lobes $\Gamma_{n_{s}, n_{i}}, n_{s} \neq n_{i}$, according to the inequality $[10,31]$

$$
\Theta_{n_{s}, n_{i}}=\frac{1}{3} \sqrt{\Gamma_{n_{s}, n_{s}} \Gamma_{n_{i}, n_{i}}}-\Gamma_{n_{s}, n_{i}}<0 .
$$

Accordingly, $\Theta_{n_{s}, n_{i}}>0$ corresponds to spatial correlations that cannot be generated by classical means. We analyze the nonclassicality of generated states and compare it to the nonclassicality obtained from theory. In the main manuscript, we present total nonclassicality summed over all waveguide numbers, while in the Supplemental Material, we show individual nonclassicalities for different waveguide combinations [32].

We note that for a separable state of two degenerate photons, the correlations have a form of $\Gamma_{n_{s}, n_{i}}=\left|\phi_{n_{s}} \phi_{n_{i}}\right|^{2}$, such that the nonclassicality inequality (G1) is never violated. Indeed, positive nonclassicality can only be registered for nonseparable entangled photon states.

[1] J. P. Dowling, Schrodinger's Killer App: Race to Build the World's First Quantum Computer (Taylor and Francis, New York, 2013).

[2] B. P. Lanyon, M. Barbieri, M. P. Almeida, T. Jennewein, T. C. Ralph, K. J. Resch, G. J. Pryde, J. L. O'Brien, A. Gilchrist, and A. G. White, Simplifying Quantum Logic Using Higher-Dimensional Hilbert Spaces, Nat. Phys. 5, 134 (2009).

[3] J. I. Cirac and P. Zoller, Quantum Computations with Cold Trapped Ions, Phys. Rev. Lett. 74, 4091 (1995).

[4] C. F. Roos, M. Riebe, H. Haffner, W. Hansel, J. Benhelm, G. P. T. Lancaster, C. Becher, F. Schmidt-Kaler, and R. Blatt, Control and Measurement of Three-Qubit Entangled States, Science 304, 1478 (2004).

[5] E. Knill, R. Laflamme, and G. J. Milburn, A Scheme for Efficient Quantum Computation with Linear Optics, Nature (London) 409, 46 (2001).

[6] C. Schuck, G. Huber, C. Kurtsiefer, and H. Weinfurter, Complete Deterministic Linear Optics Bell State Analysis, Phys. Rev. Lett. 96, 190501 (2006).

[7] M. N. O'Sullivan-Hale, I. Ali Khan, R. W. Boyd, and J. C. Howell, Pixel Entanglement: Experimental Realization of Optically Entangled Qudits, Phys. Rev. Lett. 94, 220501 (2005).

[8] M. Zukowski, A. Zeilinger, and M. A. Horne, Realizable Higher-Dimensional Two-Particle Entanglements via Multiport Beam Splitters, Phys. Rev. A 55, 2564 (1997).

[9] A. Rossi, G. Vallone, A. Chiuri, F. De Martini, and P. Mataloni, Multipath Entanglement of Two Photons, Phys. Rev. Lett. 102, 153902 (2009).

[10] A. Peruzzo, M. Lobino, J. C. F. Matthews, N. Matsuda, A. Politi, K. Poulios, X. Q. Zhou, Y. Lahini, N. Ismail, K. Worhoff, Y. Bromberg, Y. Silberberg, M. G. Thompson, 
and J. L. O'Brien, Quantum Walks of Correlated Photons, Science 329, 1500 (2010).

[11] J. O. Owens, M. A. Broome, D. N. Biggerstaff, M.E. Goggin, A. Fedrizzi, T. Linjordet, M. Ams, G. D. Marshall, J. Twamley, M. J. Withford, and A. G. White, Two-Photon Quantum Walks in an Elliptical Direct-Write Waveguide Array, New J. Phys. 13, 075003 (2011).

[12] A. Schreiber, A. Gabris, P. P. Rohde, K. Laiho, M. Stefanak, V. Potocek, C. Hamilton, I. Jex, and C. Silberhorn, A 2D Quantum Walk Simulation of Two-Particle Dynamics, Science 336, 55 (2012).

[13] B. J. Metcalf, N. Thomas-Peter, J. B. Spring, D. Kundys, M. A. Broome, P. C. Humphreys, X. M. Jin, M. Barbieri, W. S. Kolthammer, J. C. Gates, B. J. Smith, N. K. Langford, P. G. R. Smith, and I. A. Walmsley, Multiphoton Quantum Interference in a Multiport Integrated Photonic Device, Nat. Commun. 4, 1356 (2013).

[14] G. Di Giuseppe, L. Martin, A. Perez-Leija, R. Keil, F. Dreisow, S. Nolte, A. Szameit, A. F. Abouraddy, D. N. Christodoulides, and B.E. A. Saleh, Einstein-PodolskyRosen Spatial Entanglement in Ordered and Anderson Photonic Lattices, Phys. Rev. Lett. 110, 150503 (2013).

[15] K. Poulios, R. Keil, D. Fry, A. Meinecke, Jasmin D. F. Matthews, Jonathan C. A. Politi, M. Lobino, M. Gräfe, M. Heinrich, S. Nolte, A. Szameit, and J. L. O'Brien, Quantum Walks of Correlated Photon Pairs in Two-Dimensional Waveguide Arrays, Phys. Rev. Lett. 112, 143604 (2014).

[16] S. S. Straupe, D. P. Ivanov, A. A. Kalinkin, I. B. Bobrov, and S. P. Kulik, Angular Schmidt Modes in Spontaneous Parametric Down-Conversion, Phys. Rev. A 83, 060302 (2011).

[17] A. B. U'Ren, C. Silberhorn, K. Banaszek, I. A. Walmsley, R. Erdmann, W. P. Grice, and M. G. Raymer, Generation of Pure-State Single-Photon Wavepackets by Conditional Preparation Based on Spontaneous Parametric Downconversion, Laser Phys. 15, 146 (2005).

[18] R. Iwanow, R. Schiek, G. Stegeman, T. Pertsch, F. Lederer, Y. Min, and W. Sohler, Arrays of Weakly Coupled, Periodically Poled Lithium Niobate Waveguides: Beam Propagation and Discrete Spatial Quadratic Solitons, Optoelectron. Rev. 13, 113 (2005).

[19] A. S. Solntsev, A. A. Sukhorukov, D. N. Neshev, and Y. S. Kivshar, Spontaneous Parametric Down-Conversion and Quantum Walks in Arrays of Quadratic Nonlinear Waveguides, Phys. Rev. Lett. 108, 023601 (2012).

[20] H. Schmitz, R. Matjeschk, C. Schneider, J. Glueckert, M. Enderlein, T. Huber, and T. Schaetz, Quantum Walk of a Trapped Ion in Phase Space, Phys. Rev. Lett. 103, 090504 (2009).

[21] M. Karski, L. Forster, J. M. Choi, A. Steffen, W. Alt, D. Meschede, and A. Widera, Quantum Walk in Position Space with Single Optically Trapped Atoms, Science 325, 174 (2009).

[22] J. C. F. Matthews and M. G. Thompson, Quantum Optics: An Entangled Walk of Photons, Nature (London) 484, 47 (2012).

[23] A. Schreiber, K. N. Cassemiro, V. Potocek, A. Gabris, P. J. Mosley, E. Andersson, I. Jex, and C. Silberhorn, Photons Walking the Line: A Quantum Walk with Adjustable Coin Operations, Phys. Rev. Lett. 104, 050502 (2010).
[24] L. Sansoni, F. Sciarrino, G. Vallone, P. Mataloni, A. Crespi, R. Ramponi, and R. Osellame, Polarization Entangled State Measurement on a Chip, Phys. Rev. Lett. 105, 200503 (2010).

[25] T. Kitagawa, M. A. Broome, A. Fedrizzi, M. S. Rudner, E. Berg, I. Kassal, A. Aspuru-Guzik, E. Demler, and A. G. White, Observation of Topologically Protected Bound States in Photonic Quantum Walks, Nat. Commun. 3, 882 (2012).

[26] D. N. Christodoulides, F. Lederer, and Y. Silberberg, Discretizing Light Behaviour in Linear and Nonlinear Waveguide Lattices, Nature (London) 424, 817 (2003).

[27] R. Kruse, F. Katzschmann, A. Christ, A. Schreiber, S. Wilhelm, K. Laiho, A. Gabris, C. S. Hamilton, I. Jex, and C. Silberhorn, Spatio-spectral Characteristics of Parametric Down-Conversion in Waveguide Arrays, New J. Phys. 15, 083046 (2013).

[28] S. Longhi, Quantum-Optical Analogies Using Photonic Structures, Laser Photonics Rev. 3, 243 (2009).

[29] I. L. Garanovich, S. Longhi, A. A. Sukhorukov, and Y. S. Kivshar, Light Propagation and Localization in Modulated Photonic Lattices and Waveguides, Phys. Rep. 518, 1 (2012).

[30] M. Grafe, A. S. Solntsev, R. Keil, A. A. Sukhorukov, M. Heinrich, A. Tunnermann, S. Nolte, A. Szameit, and Y. S. Kivshar, Biphoton Generation in Quadratic Waveguide Arrays: A Classical Optical Simulation, Sci. Rep. 2, 562 (2012).

[31] Y. Bromberg, Y. Lahini, R. Morandotti, and Y. Silberberg, Quantum and Classical Correlations in Waveguide Lattices, Phys. Rev. Lett. 102, 253904 (2009).

[32] See Supplemental Material at http://link.aps.org/ supplemental/10.1103/PhysRevX.4.031007 for periodically poled waveguide array characterization, comparison between experimental and theoretical results, experimental results obtained in a waveguide array without periodic poling, and an estimate of the experimental entanglement lower bound.

[33] J. D. Jackson, Classical Electrodynamics (Wiley, New York, 1998).

[34] X.-C. Yao, T.-X. Wang, H.-Z. Chen, W.-B. Gao, A. G. Fowler, R. Raussendorf, Z.-B. Chen, N.-L. Liu, C.-Y. Lu, Y.-J. Deng, Y.-A. Chen, and J.-W. Pan, Experimental Demonstration of Topological Error Correction, Nature (London) 482, 489 (2012).

[35] S. Yokoyama, R. Ukai, S. C. Armstrong, C. Sornphiphatphong, T. Kaji, S. Suzuki, J.-i. Yoshikawa, H. Yonezawa, N. C. Menicucci, and A. Furusawa, Ultra-Large-Scale Continuous-Variable Cluster States Multiplexed in the Time Domain, Nat. Photonics 7, 982 (2013).

[36] A. S. Solntsev, A. A. Sukhorukov, D. N. Neshev, and Y.S. Kivshar, Photon-Pair Generation in Arrays of Cubic Nonlinear Waveguides, Opt. Express 20, 27441 (2012).

[37] K. V. Lewis-Swan and R. J. Kheruntsyan, Proposal for Demonstrating the Hong-Ou-Mandel Effect with Matter Waves, Nat. Commun. 5, 3752 (2014).

[38] O. Morsch and M. Oberthaler, Dynamics of Bose-Einstein Condensates in Optical Lattices, Rev. Mod. Phys. 78, 179 (2006). 
[39] F. Lederer, G. I. Stegeman, D. N. Christodoulides, G. Assanto, M. Segev, and Y. Silberberg, Discrete Solitons in Optics, Phys. Rep. 463, 1 (2008).

[40] W. P. Grice and I. A. Walmsley, Spectral Information and Distinguishability in Type-II Down-Conversion with a Broadband Pump, Phys. Rev. A 56, 1627 (1997).
[41] G. J. Edwards and M. Lawrence, A Temperature-Dependent Dispersion-Equation for Congruently Grown LithiumNiobate, Opt. Quantum Electron. 16, 373 (1984).

[42] A. Ekert and P. L. Knight, Entangled Quantum-Systems and the Schmidt Decomposition, Am. J. Phys. 63, 415 (1995). 